\title{
Modelo Pedagógico ML-SAI: Reflexões Sobre o Uso de Recursos Tecnológicos Durante a Pandemia de Covid-19
}

Ernane Rosa Martins, IFG, Brasil, ernane.martins@ifg.edu.br, https://orcid.org/00000002-1543-1108

Luís Manuel Borges Gouveia, UFP, Portugal, lmbg@ufp.edu.pt, https://orcid.org/00000002-2079-3234

Resumo. $O$ modelo pedagógico ML-SAI visa orientar atividades de m-learning, por meio da metodologia Ativa da Sala de Aula Invertida (SAI). Este artigo tem como objetivo relatar a aplicação do ML-SAI durante a pandemia de Covid-19 e promover algumas reflexões sobre o uso dos recursos tecnológicos envolvidos. Para tal, foi realizada um estudo de caso, tendo como instrumento de coleta de dados: observação, questionários on-line e o registro no ambiente virtual Moodle. Os resultados demonstraram que embora a utilização do modelo exija maior planejamento por parte dos professores, os benefícios alcançados são muito interessantes, tanto para os alunos, quanto para os professores, indicando uma receptividade favorável ao modelo neste momento de enormes desafios causados pela pandemia de Covid-19.

Palavras-chave: Sala de Aula Invertida, M-learning, Recursos Tecnológicos.

\section{ML-SAI Pedagogical Model: Reflections on the Use of Technological Resources During the Covid-19 Pandemic}

\begin{abstract}
The ML-SAI pedagogical model aims to guide m-learning activities, using the Active Classroom Inverted (SAI) methodology. This article aims to report the application of ML-SAI during the Covid-19 pandemic and to promote some reflections on the use of the technological resources involved. To this end, a case study was carried out, using the data collection instrument: observation, online questionnaires and registration in the virtual Moodle environment. The results found showed that although the use of the model requires more planning by teachers, the benefits achieved are very interesting, both for students and teachers, indicating a favorable receptivity to the model at this time of enormous challenges caused by the pandemic of Covid-19.
\end{abstract}

Keywords: Inverted Classroom, M-learning, Technological Resources.

\section{Introdução}

A pandemia de COVID-19 apresentou novas discussões sobre Educação a Distância $(\mathrm{EaD})$ e Ensino Remoto Emergencial (ERE), por ser a única alternativa viável para assegurar a educação neste período. Assim, é importante diferenciar EaD e atividades do ERE. $\mathrm{Na} \mathrm{EaD,} \mathrm{desde} \mathrm{o} \mathrm{planejamento} \mathrm{até} \mathrm{a} \mathrm{execução} \mathrm{de} \mathrm{um} \mathrm{curso} \mathrm{ou} \mathrm{de}$ uma disciplina, há um modelo subjacente de educação que ampara as escolhas pedagógicas e organiza os processos de ensino e de aprendizagem. Existem concepções teóricas, fundamentos metodológicos e especificidades (Rodrigues, 2020). 
No ERE, por sua vez, existe uma adaptação curricular temporária, como uma alternativa para que ocorram as atividades acadêmicas relacionadas às diversas disciplinas dos cursos, devido às circunstancias de crise, esta envolve o uso de soluções de ensino totalmente remotas, que seriam ministradas presencialmente (Hodges, et. al., 2020). Assim, é notório a necessidade de investimento na formação permanente dos professores, pois precisam cada vez mais buscar o "aprender a aprender", com investigação e pesquisa, visto as mudanças no contexto da educação no Brasil e no mundo. Este momento da história, vem trazer um enorme desafio, os educadores foram forçados a adaptar-se a está nova realidade, sem modelos ou receitas prontas, ou seja, um enorme desafio, principalmente pela necessidade da utilização das tecnologias nessa nova forma de ensinar (Valente, 2020).

A Sala de Aula Invertida (SAI), conceito que fundamenta o modelo pedagógico ML-SAI, é uma metodologia ativa, em que o aluno realiza, em casa, algumas atividades que, normalmente, seriam realizadas na escola, e o tempo em sala de aula é reservado para a realização de atividades mais ativas, tais como: experimentos, debates, atividades em grupo, pesquisas, entre outros (Mattar, 2017). No ERE o modelo ML-SAI foi adaptado para que os alunos realizassem as atividades da escola em casa, e os momentos on-line, geralmente mais curtos, seriam para a realização das atividades ativas.

Conforme Martins et al. (2018) a maioria dos alunos e professores estão fazendo uso dos dispositivos móveis de comunicação para acessar diversos materiais didáticos, ambientes de interação entre alunos e professor, atividades escolares e para o compartilhamento de arquivos. Os dispositivos móveis de comunicação apresentam-se como uma alternativa viável para ampliar as possibilidades dos estudantes, proporcionando novas formas na construção e desenvolvimento do conhecimento.

Neste estudo, utiliza-se a definição de modelo pedagógico como sendo "um sistema de premissas teóricas que representa, explica e orienta a forma como se aborda 0 currículo e que se concretiza nas práticas pedagógicas e nas interações professor-alunoobjeto de conhecimento", podendo ser embasado por uma ou várias teorias de aprendizagem, ou suas reinterpretações (Behar; Passerino \& Bernardi, 2007, p. 4).

Assim, este artigo tem como objetivo relatar a aplicação do modelo pedagógico ML-SAI durante a pandemia de Covid-19 e promover algumas reflexões sobre o uso dos recursos tecnológicos envolvidos, com foco principal nos smartphones. Tendo em vista o objetivo proposto, na seção 2 é apresentada a estrutura e estratégias do ML-SAI. Na seção 3 é estabelecido o método, as técnicas e procedimentos metodológicos utilizados. $\mathrm{Na}$ seção 4, apresenta-se os resultados encontrados. Por fim, a seção 5 apresenta algumas considerações finais sobre o presente trabalho.

\section{Estrutura e Estratégias do ML-SAI}

O modelo pedagógico ML-SAI (Figura 1) foi formatado para fornecer algumas sugestões de estratégias a professores interessados em utiliza-lo, orientando estes no desenvolvimento das atividades de m-learning. No ML-SAI a Arquitetura Pedagógica (AP) foi reestruturada e fundamentada levando em consideração os conceitos da SAI, os aspectos relacionados a utilização dos dispositivos móveis e os estudos exploratórios preliminares realizados, assim, a AP foi estabelecida em seis aspectos, sendo estes: contexto, normatização, papeis, tecnologias, ações e limitações (Martins \& Gouveia, 2019b). 

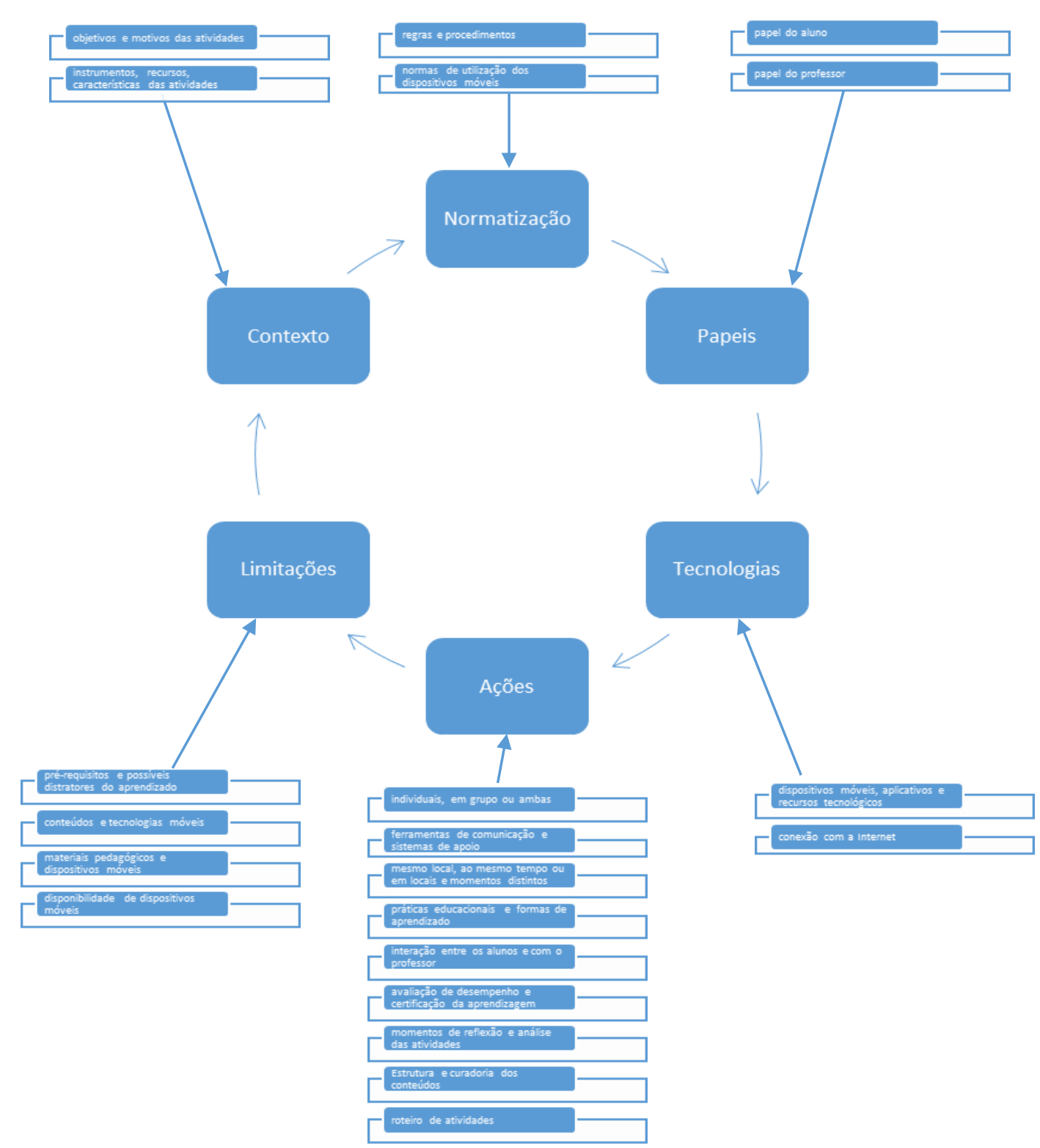

Figura 1 - Modelo pedagógico ML-SAI

Fonte: Martins \& Gouveia (2019a)

A Arquitetura Pedagógica (AP) e as estratégias para a aplicação da AP definidas para as atividades de m-learning são apresentadas detalhadamente na Tabela 1.

Tabela 1 - Modelo pedagógico ML-SAI (Martins \& Gouveia, 2019a).

\begin{tabular}{ll}
\hline \multicolumn{1}{c}{ AP } & \multicolumn{1}{c}{ Estratégias para a Aplicação da AP } \\
\hline \multirow{2}{*}{ Contexto } & $\begin{array}{l}\text { Definir os objetivos e motivos das atividades e ações proposta, deixando-os claros para todos } \\
\text { os envolvidos; } \\
\text { Identificar os instrumentos, recursos, características das atividades e ações, dos alunos e do } \\
\text { curso; }\end{array}$ \\
\hline \multirow{2}{*}{ Normatização } & $\begin{array}{l}\text { Organizar regras e procedimentos para orientar as ações e interações; } \\
\text { Estabelecer normas para utilização dos dispositivos móveis (quando utilizar, qual a } \\
\text { finalidade, etc.); }\end{array}$ \\
\hline \multirow{2}{*}{ Papeis } & $\begin{array}{l}\text { Compreender o papel do aluno no processo de aprendizagem, suas motivações, interesses e } \\
\text { habilidades; } \\
\text { Entender o papel do professor como condutor e facilitador da aprendizagem; }\end{array}$ \\
\hline Tecnologias & Definir os dispositivos móveis, aplicativos e recursos tecnológicos que serão utilizados,
\end{tabular}


considerando as características físicas, técnicas e funcionais dos mesmos, tais como: ambiente virtual, Sílabe, Moodle, Facebook, Khan Academy, YouTube, vídeo-aula, músicas, slides, fotografias, áudios, textos, entre outros, estabelecendo prioridade para aplicativos livres e gratuitos;

Verificar a necessidade e disponibilidade de conexão com a Internet;

Especificar se as ações serão individuais, em grupo ou ambas, se estas serão comuns a todos os alunos ou diferenciadas por aluno ou grupo de alunos;

Definir ferramentas de comunicação e sistemas de apoio para dar suporte aos alunos em caso de dificuldades;

Definir se as ações serão realizadas em um mesmo local, ao mesmo tempo ou em locais e momentos distintos;

Estabelecer práticas educacionais favoráveis ao aprendizado (situações problemas, aplicações práticas, colaborativas, autônomas, críticas, em contextos reais, pesquisas), levando em consideração os ambientes de aprendizagem (on-line, salas de aula, laboratórios) de preferência com os dispositivos móveis dos próprios alunos;

Incentivar a interação entre os alunos e com o professor, por meio do uso de dispositivos móveis, com foco no desenvolvimento da atividade proposta;

Determinar os mecanismos de avaliação de desempenho e certificação da aprendizagem, se

Ações individuais ou em equipes, de preferência continua, e disponibiliza-los para os alunos;

Estabelecer momentos de reflexão e análise das atividades realizadas, buscando colaborar na melhoria continua de novas atividades;

Estruturar os conteúdos que serão disponibilizados em ambiente virtual, para que os alunos possam acessa-los por meio de um dispositivo móvel, quando e quantas vezes quiserem, se possível com o acompanhamento das visualizações pelo professor;

Realizar uma curadoria dos conteúdos já existentes na Internet, por meio de plataformas como Khan Academy e o YouTube em busca de bons vídeos educativos, ou caso não sejam encontrados, gravar vídeos ou áudios utilizando as ferramentas que existem no próprio dispositivo móvel;

Estimular diferentes formas de aprendizado por meio de diferentes fontes de conteúdo, tais como: vídeos, áudios, imagens, textos, slides, questões, entre outras;

Elaborar um roteiro de atividades do que será feito dentro da sala de aula, de modo a otimizar o tempo em sala de aula, utilizando projetos, trabalhos ou solução de problemas, que se conectem com o que foi visto previamente na plataforma;

Levantar os principais pré-requisitos das atividades e possíveis distratores do aprendizado;

Identificar quais conteúdos podem ser melhor trabalhados com tecnologias móveis;

Verificar se os materiais pedagógicos podem ser utilizados em dispositivos móveis, Limitações considerando tamanho da tela, usabilidade, capacidade de armazenamento e modelos de dispositivos diferentes;

Verificar a disponibilidade de dispositivos móveis, tomadas para recarregar as baterias dos celulares, conexão com a Internet, quando necessário, e se os aplicativos apresentam interface adequada a aprendizagem do conteúdo.

O ML-SAI foi concebido para colaborar com a realização de diversas atividades de m-learning, com diferentes conteúdos em dispositivos móveis.

\section{Metodologia}

O método adotado para a realização desta pesquisa foi o estudo de caso, escolhido por ser de natureza empírica que investiga um determinado fenômeno, geralmente contemporâneo, dentro de um contexto real de vida, tratando-se de uma análise aprofundada de um ou mais objetos (casos), permitindo seu amplo e detalhado conhecimento (Gil, 2002). Este tem como objetivo, aprofundar o conhecimento acerca de um problema não suficientemente definido, visando estimular a compreensão, sugerir hipóteses e questões ou simplesmente desenvolver a teoria. Está presente pesquisa pode ser classificada ainda como exploratória, de cunho quantitativa e qualitativa, pois visa desenvolver, esclarecer ou modificar conceitos e ideias sobre determinado assunto (Gil, 1999). Este estudo, busca apresentar soluções, as exigências da atualidade diante da V. $19 \mathrm{~N}^{\mathrm{o}} 1$, julho, 2021 DOI: https://doi.org/10.22456/1679-1916.118538 RENOTE 
pandemia da COVID 19 que assola o mundo inteiro, por meio de uma análise crítica e reflexiva acerca das mudanças vivenciadas neste novo contexto da prática docente, que exige a formação permanente, do uso das tecnologias de informação e comunicação para possibilitar a efetivação do processo de ensino-aprendizagem de forma remota e o enfrentamento eficaz dos desafios impostos por essa nova realidade.

Foram utilizados como instrumentos de coleta de dados a observação realizada pelo pesquisador de forma não estruturada, as percepções dos alunos e professores envolvidos, recolhidas por meio do questionário on-line e ainda mediante dos registros do ambiente virtual Moodle. O modelo ML-SAI foi utilizado nas atividades remotas durante o primeiro semestre de 2021, no curso superior noturno de Sistemas de Informação (SI), com a participação de 40 alunos, de três disciplinas, sendo elas: Estrutura de dados I, Padrões de projeto e Sistemas de computação aplicados. O professor pesquisador ministrou as disciplinas, optando pela utilização dos dispositivos móveis dos próprios alunos, para permitir maior aproximação com o que os alunos estão habituados a utilizar em seu dia a dia e facilitar a aprendizagem. $\mathrm{O}$ ambiente de aprendizagem on-line adotado foi o Moodle, escolhido por já ser utilizado pelos alunos.

O questionário utilizado neste estudo foi adaptado do que foi empregado no doutorado do professor pesquisador. As principais questões que integraram o questionário foram: Os participantes possuíam smartphone, qual o tipo e recursos dos mesmos? Os participantes utilizavam o smartphone para estudo? Os dispositivos móveis colaboram para fins educativos? O uso de diversos recursos tecnológicos no apoio à disciplina contribuiu para a aprendizagem? Os smartphones foram recursos importantes para a aprendizagem? Os aplicativos dos smartphones utilizados foram fáceis de usar? A proposta de uso do smartphone, foi importante para a disciplina? Foram utilizados recursos tecnológicos adequados? Apresente alguns comentários que considere relevante? Ao final, foi realizada uma análise crítica dos dados recolhidos no questionário aplicado aos alunos, com a organização, cruzamento e comparação das informações similares por contextos.

\section{Resultados}

Quando perguntados se os participantes possuíam smartphone, qual o tipo e recursos dos mesmos? Todos os estudantes participantes afirmaram que possuem smartphone, com acesso à Internet e aplicativos digitais. Quando perguntados qual o modelo de smartphone os alunos possuíam, buscou-se saber se algum modelo de smartphone poderia comprometer a utilização do modelo ML-SAI. Os estudantes relataram que possuem diversos tipos de aparelhos, tais como: Redmi Note 8, iPhone, Moto g9 play, Iphone 7, LX-Stile, Xiaomi Red Note 8, OnePlus 6T, Alcatel, Noye 9, Galaxy a6+, lg k50s, Poco X3, Iphone 6s, Xiaomi Redmi Note 8, Sistema Android, Galaxy $s 9$. Todos os aparelhos apresentam condições de serem utilizados para fins educacionais durante a pandemia com o modelo pedagógico ML-SAI.

Sobre os recursos dos aparelhos utilizados, pretendia-se saber quais os recursos dos smartphones favorecem a utilização do modelo pedagógico ML-SAI. Entre os recursos citados estão: android, Zoom, Meet, Notion, Trello, Email, Internet, modem ap, ancoragem, dex station, mensagens, ligações, consumo de vídeos, estudos, jogos, Acesso à Internet e uso de apps, Mensagens e pesquisas, Google meet, google drive, redes sociais e estudos. 
Perguntados se os estudantes já utilizavam os smartphones para o estudo? Nesta pergunta pretendia-se saber se os estudantes utilizam seu smartphone no estudo antes da pandemia. Os alunos responderam que já utilizaram os aparelhos em diversas disciplinas em seus cursos e de várias formas possíveis, tais como: "Sim, como minha jornada em ônibus é relativamente longa o utilizo para estudos, então é uma ferramenta essencial nesses momentos", "Sim, nas disciplinas da faculdade e por vontade própria e o uso do smartphone foi significativo pois é rápido e portátil ou seja você tem sempre informação com você", "Sim, para estudar pra faculdade, para ler livros", "Sim, nas pesquisas e visualização de conteúdos", "Sim, para realização de cursos externos. O uso do aparelho permite acessar as aulas de qualquer lugar praticamente, contanto que se tenha conexão com a Internet", "Sim, banco de dados, sistemas de computação aplicados a tomada de decisão. Foi significativo sim, facilidade de uso", "Sim. Para pesquisas e desenvolvimento de projeto", "Sim, utilizei o duolingo", "Sim, utilizei para pesquisas e para as aulas remotas", "Sim, não tenho preferência em estudar por smartphone, vejo como contingência", "Sim, em alguns momentos usei nas aulas quando não podia utilizar o computador. Foi muito útil", "Sim, já utilizei alguns aplicativos para uso educacional, como: Edmodo". Ninguém respondeu que nunca utilizou o smartphone para estudos.

$\mathrm{Na}$ pergunta, os dispositivos móveis colaboram para fins educativos? Nesta pergunta pretendia-se saber a opinião dos alunos em relação ao uso dos dispositivos móveis para fins educativos. Todos os estudantes afirmaram que os dispositivos móveis quando utilizados no ensino poderiam colaborar para diminuir as dificuldades de aprendizagem, facilitando o acesso aos materiais educacionais e a comunicação entre eles.

Perguntados se o uso de diversos recursos tecnológicos no apoio à disciplina contribuiu para a aprendizagem? Nesta pergunta buscou-se saber a opinião dos estudantes em relação aos recursos tecnológicos como apoio à disciplina. As respostas foram todas que sim, sendo muito elogiada principalmente por facilitar o aprendizado dos conteúdos.

Questionou-se também se os smartphones foram recursos importantes para a aprendizagem? Nesta pergunta pretendia-se saber a opinião dos alunos em relação a importância dos smartphones na aprendizagem. A grande maioria respondeu: "Sim, com certeza e cada vez mais vão ser usados como recursos para aprendizado", "Sem dúvida", "sim, parcialmente", "só utilizo smartphone para estudo na falta de PC", "Sim, mas não é o principal recurso".

Os alunos apreciaram muito a utilização de aplicativos de mensagens instantâneas dos smartphones, principalmente por permitir retirar as dúvidas mais rapidamente. A fácil utilização dos mesmos, principalmente por já estarem familiarizados com sua utilização também foi um ponto elogiado pelos estudantes.

A utilização dos smartphones, foi importante para a disciplina? Nesta pergunta pretendia-se saber a opinião dos alunos em relação ao uso dos smartphones na disciplina. Em quase sua totalidade, os alunos responderam que sim, com apenas um que disse "mais ou menos" e outro que disse "Nem tanto". Demostrando que na opinião da maioria dos respondentes, a proposta do uso do smartphone foi importante para a disciplina. 
Alguns alunos relataram que o fato de realizarem os estudos com antecedência, as aulas síncronas ficam mais produtivas. Alguns alunos comentaram que as aulas síncronas poderiam ser maiores, pois não havia tempo para evoluir muito nos conteúdos apresentados. Os alunos relataram que suas participações foram serias e comprometidas, realizando todas as tarefas antes das aulas síncronas.

Relataram ainda, que a ajuda dos colegas neste momento atípico, foi ainda mais importante para a sua aprendizagem, disse: "ainda mais com o ensino on-line a opinião e a ajuda de todos faz com que tenhamos outras visões sobre o assunto". Responderam que a atuação do professor foi extremante importante para sua aprendizagem. Sobre a utilização de recursos tecnológicos adequados, a maioria dos alunos afirmaram que sim, apesar de não especificarem de que forma. Alguns relataram que diante da atual situação em que estamos vivendo, consideram importantes as atividades das aulas não presenciais/remotas, como por exemplo: "muito importante assim não ficamos sem aula", e "Com certeza, não podemos ficar parados".

De maneira geral, consideraram que a quantidade de atividades enviadas pelo professor foi adequada, um disse que: "Melhor qualidade do que quantidade", e outro ainda afirmou que "Depende do nível das aulas assíncronas, não adianta passar muitos exercícios se não tem como discutir estes exercícios na aula síncrona".

Entre as principais dificuldades relatadas pelos alunos estão: "Um pouco por falta de tempo mesmo e as vezes muitas atividades de disciplinas diferentes", "Falta de exemplos", "Em meio a pandemia quem trabalha com tecnologia teve o trabalho reformulado, então no início foi bem complicado. Além do mais, meus horários foram reajustados, entrando mais tarde e saindo mais tarde do trabalho, comprometendo na presença da aula on-line", e "Tive dificuldade em fazer as primeiras tarefas, mas os seguintes foram mais fáceis de fazer".

Sobre as atividades realizadas virtualmente, e o contato com o professor. Responderam que: "Bem satisfatório", "Bom, toda semana nos reunimos e temos a oportunidade de discutir sobre o tema proposto", "Razoável", "Atualmente foi pouco", "Através de e-mails e grupos WhatsApp", "Pouco ou nenhum, em 45 minutos não se dá para evoluir muito", "Distante", "Ótima", "Através do email, moodle ou redes sociais", e "Somente nas aulas on-line".

Perguntados o que poderia melhorar? Estes responderam que: "Sendo aplicado o conteúdo que foi programado", "Alguns professores precisam dar mais atenção aos alunos e não deixar de dar feedback", "Mais tempo de aula síncrona", "Mais exemplos dos professores", "Agilidade nas respostas" e "Está bom".

A respeito de que acreditam que este modelo não presencial é suficiente para a aprendizagem, responderam que: "Acredito, mas depende mais do aluno", "Sim, salvo em casos onde, o recurso do aluno dispõe não seja o suficiente para o modelo", "Totalmente não, mas supre a necessidade", "Até agora sim", e "É um modelo mais difícil, porém necessário".

Perguntados se acreditam que os professores estão preparados para ensinar de forma remota. Responderam que: "Alguns", "Talvez", "Todos estão se adaptando, mas estão se preparando cada vez mais", "A maioria sim", "Mais ou menos", e "Nem os professores estão prontos para ministrar aulas a distância, como tão pouco os alunos estão preparados. Mas a adaptação virá com o tempo". 
Sobre quais são as principais dificuldades das atividades não presenciais? Responderam: "Nenhuma", "A Internet com oscilações em determinados dias", "As falhas de conexão com a Internet", "Ter foco, conteúdo não é suficiente", "Contra tempos como queda de conexão", "alunos que tem dúvida, podem ter dificuldade de saná-las", "O tempo da aula síncrona ser muito curto", "Compreensão dos professores", "Em alguns casos encontrar conteúdos para estudo e em outros agilidade dos professores em postar o conteúdo, visto que em alguns casos os professores demoraram a postar materiais de estudo e atividades, reduzindo o tempo útil de execução das mesmas", "No começo o problema foi minha Internet, mas já resolvi esse problema", e "Disciplina para realizar os estudos".

Sobre quais são os principais entraves para estudar em casa? Foram citados: "Encontrar um ambiente calmo para estudos", "O comodismo e a Internet", "Foco", "Falta de atenção, barulho", "disponibilidade de horário", "recursos (exemplo computadores) para disciplinas praticas", "Nenhum", "Eu não tenho nenhum, meu único problema é com o tempo da aula síncrona", "Praticidade, foco e gestão de tempo", "Barulho", e "Tempo, família e disciplina".

Por fim, foi solicitado aos alunos que apresentassem, alguns comentários, de modo a entender as opiniões gerais dos alunos de forma livre. Assim, responderam que: "As aulas remotas deveriam ter um maior tempo para as matérias não ficarem tão corridas e melhorar no ensino, e foi uma boa experiência metodologia ativa", "A metodologia utilizada foi boa por compartilhar as opiniões gerando assim várias visões sobre certo assunto da tecnologia", "Tem sido bom estudar em casa e sinto que aprendo mais", "Em adequação nos ambientes, mas buscando sempre atender com os recursos disponíveis", "Legal a proposta, devem melhorar cada vez mais a participação de todos nesse quesito de ensino a distância pois é algo um pouco complicado do que aulas presenciais", "O tempo de aula foi curto devido alguns contra tempos", "A estratégia adotada, é muito boa, é de fácil assimilação e aprendizado", "Seria melhor se as aulas síncronas fossem de duração maior, 45 minutos é pouco para tanto conteúdo", "Em geral, os professores, mesmo com o tempo reduzido, foram proativos", "Tudo saiu nos conformes", "Foi uma boa disciplina ministrada", "As metodologias utilizadas foram boas", "Melhor preparação de alguns professores, alguns vão dar aula sem ter algo preparado", "Mais tempo de aula síncrona", "Ao invés de trabalhos escritos ou provas, seria ideal a realização de atividades práticas, na minha visão, funciona melhor para o aprendizado à distância. Pois, ou o aluno faz ou não faz, sem meio termo", "Um planejamento mais robusto, e mais tempo de aula para as aulas não ficarem corridas", e "Disponibilização de mais conteúdo para buscarmos mais conhecimento". Alguns alunos solicitaram mais aulas gravadas, pois poderiam assistir posteriormente, definindo em qual horário assistir. Os benefícios encontrados após a utilização do modelo pedagógico ML-SAI, durante a pandemia de Covid-19, foram sintetizados e apresentados na Tabela 2.

Tabela 2 - Benefícios encontrados com a utilização do modelo pedagógico ML-

SAI, durante a pandemia de Covid-19

\begin{tabular}{|c|c|}
\hline Benefícios encontrados & Principais Depoimentos dos alunos \\
\hline \begin{tabular}{llr}
\multicolumn{3}{l}{ Possuem smartphone, com } \\
acesso à & Internet e \\
condições & de $r$ serem \\
utilizados & para & fins
\end{tabular} & $\begin{array}{l}\text { "Sim, como minha jornada em ônibus é relativamente longa o utilizo para } \\
\text { estudos então é uma ferramenta essencial nesses momentos"; } \\
\text { "Sim, nas disciplinas da faculdade e por vontade própria e o uso do smartphone } \\
\text { foi significativo pois é rápido e portátil ou seja você tem sempre informação com }\end{array}$ \\
\hline
\end{tabular}


educacionais durante a você";

pandemia, com o modelo "Sim, para estudar pra faculdade, para ler livros";

pedagógico ML-SAI.

"Sim, nas pesquisas e visualização de conteúdos";

"Sim, para realização de cursos externos. O uso do aparelho permite acessar as aulas de qualquer lugar praticamente, contanto que se tenha conexão com a Internet";

"Sim, Utilizei para pesquisas e para as aulas remotas";

"Sim. Em alguns momentos usei nas aulas quando não podia utilizar o computador. Foi muito útil";

"Sim, com certeza e cada vez mais vão ser usados como recursos para

Os smartphones foram recursos importantes para a aprendizagem.

aprendizado";

"Sem dúvida";

"Sim, parcialmente";

"Só utilizo smartphone para estudo na falta de PC";

"Sim, mas não é o principal recurso".

"Acredito, mas depende mais do aluno";

"Sim, salvo em casos onde, o recurso do aluno dispõe não seja o suficiente para

O modelo não presencial é suficiente para a o modelo";

aprendizagem "Totalmente não, mas supre a necessidade";

"Até agora sim";

"É um modelo mais difícil, porém necessário".

Os professores estão se preparando e esforçando para ensinar de forma

"Todos estão se adaptando, mas estão se preparando cada vez mais";

"A maioria sim"; remota.

"Nem os professores estão prontos para ministrar aulas a distância, como tão pouco os alunos estão preparados. Mas a adaptação virá com o tempo".

"As aulas remotas deveriam ter um maior tempo para as matérias não ficarem tão corridas e melhorar no ensino, e foi uma boa experiência metodologia ativa";

"A metodologia utilizada foi boa por compartilhar as opiniões gerando assim várias visões sobre certo assunto da tecnologia";

"Tem sido bom estudar em casa e sinto que aprendo mais";

"Em adequação nos ambientes, mas buscando sempre atender com os recursos disponíveis";

As opiniões gerais dos alunos foram positivas.
"Legal a proposta, devem melhorar cada vez mais a participação de todos nesse quesito de ensino a distância pois é algo um pouco complicado do que aulas presenciais";

"A estratégia adotada, é muito boa, é de fácil assimilação e aprendizado";

"Em geral, os professores, mesmo com o tempo reduzido, foram proativos";

"As metodologias utilizadas foram boas";

"Ao invés de trabalhos escritos ou provas, seria ideal a realização de atividades práticas, na minha visão, funciona melhor para o aprendizado à distância. Pois, ou o aluno faz ou não faz, sem meio termo";

"Disponibilização de mais conteúdo para buscarmos mais conhecimento".

\section{Considerações Finais}

Este artigo teve como objetivo relatar a aplicação do ML-SAI durante a pandemia de Covid-19 e promover algumas reflexões sobre o uso dos recursos tecnológicos envolvidos, com foco principal nos smartphones. A educação passou por um grande impacto, por causa da pandemia de COVI-19, obrigando que toda a comunidade acadêmica buscasse novas formas de lidar com as adequações do ensino. $\mathrm{O}$ ERE tornou-se a única alternativa viável neste momento de crise, buscando adequar a novas formas de ensinar, modificando as práticas docentes, experimentando, e inserindo novas tecnologias como protagonista, apesar dos enormes desafios, visto as necessidades de qualificação dos docentes e desigualdade de inclusão dos estudantes.

Portanto, neste contexto de pandemia, faz-se necessário utilizar novas estratégias de ensino e de aprendizagem, que envolvam as tecnologias da informação, visando V. $19 \mathrm{~N}^{\mathrm{o}} 1$, julho, 2021 DOI: https://doi.org/10.22456/1679-1916.118538 RENOTE 
minimizar os prejuízos e superar os desafios do ensino neste momento tão atípico. Assim, o ML-SAI surge como uma possibilidade de contribuir para inserção das tecnologias da informação na educação.

Deste modo, este trabalho verificou que embora a utilização do modelo exija maior planejamento por parte dos professores, o que foi relatado pelo professor pesquisador, os benefícios alcançados são muito interessantes tanto para os alunos quanto para os professores, indicando uma receptividade favorável ao modelo neste momento de enormes desafios causados pela pandemia de Covid-19. Facilitando a adaptação dos alunos, neste momento da história, em que vivemos está nova realidade de utilização forçada das tecnologias digitais, modificando totalmente a forma tradicional de ensino. Os alunos em sua maioria parecem entender que o momento é anormal e cheio de dificuldades, tanto para eles, como para os professores. Pretende-se realizar novas experimentações mais abrangentes, de modo a validar o uso do ML-SAI durante a Pandemia de Covid-19.

\section{Referências Bibliográficas}

BEHAR, P. A., PASSERINO, L., \& BERNARDI, M. Modelos Pedagógicos para Educação a Distância: pressupostos teóricos para a construção de objetos de aprendizagem. Revista Novas Tecnologias na Educação, Porto Alegre, v.5, n. 2, p. 1-12, 2007.

GIL, A. C. Como elaborar projetos de pesquisa. 4. Ed. São Paulo: Atlas, 2002.

GIL, A. C. Métodos e técnicas de pesquisa social. São Paulo: Atlas, 1999.

HOdGES, C, MOORE, S, LOCKEE, B, TRUST, T \& BOND, A. The Difference Between Emergency Remote Teaching and Online Learning. 2020. Disponível em: $<$ https://er.educause.edu/articles/2020/3/the-difference-between-emergency-remoteteaching-and-online-learning $>$

MARTINS, E. R., GERALDES, W. B., AFONSECA, U. R., \& GOUVEIA, L. M. B. Tecnologias Móveis em Contexto Educativo: uma Revisão Sistemática da Literatura. Revista Novas Tecnologias Na Educação, v. 16, n. 1, p. 1-10, 2018.

MARTINS, E. R.; GOUVEIA, L. M. B. M-Learning e Sala de Aula Invertida: Construção de um Modelo Pedagógico (ML-SAI). In: Solange Aparecida de Souza Monteiro. (Org.). Inquietações e proposituras na formação docente. 1ed. Ponta Grossa, PR: Atena Editora, v. 1, p. 184-192, 2019a.

MARTINS, E. R.; GOUVEIA, L. M. B. Modelo Pedagógico de M-Learning em Sala de Aula Invertida (MLSAI): Reflexões Sobre o Uso de Recursos Tecnológicos. Revista novas tecnologias na educação, v. 17, p. 407-416, $2019 \mathrm{~b}$.

MATTAR, J. Metodologias Ativas: para a educação presencial, blended e a distância. 1 ed. São Paulo: Artesanato Educacional, 118p, 2017.

RODRIGUES, A. Ensino remoto na Educação Superior: desafios e conquistas em tempos de pandemia. 2020. SBC Horizontes, jun. ISSN 2175-9235. Disponível em: $<$ http://horizontes.sbc.org.br/index.php/2020/06/17/ensino-remoto-na-educacao-superior/>.

VALENTE, G. S. C et. al. O ensino remoto frente às exigências do contexto de pandemia: Reflexões sobre a prática docente. Research, Society and Development, v. 9, n. 9, 2020. Disponível em: <https://rsdjournal.org/index.php/rsd/article/view/ 8153/7109>. 